\title{
ON ENTIRE FUNCTIONS WHICH SHARE ONE SMALL FUNCTION CM WITH THEIR FIRST DERIVATIVE
}

\author{
Amer H. H. AL-Khaladi
}

\begin{abstract}
The paper generalizes a result of R. Brück and makes an example which shows that the generalization is precise.
\end{abstract}

\section{Introduction and results}

In this paper the term "meromorphic" will always mean meromorphic in the complex plane. We use the standard notations and results of the Nevanlinna theory (See [2] or [3], for example). In particular, $S(r, f)$ denotes any quantity satisfying $S(r, f)=o(T(r, f))$ as $r \rightarrow \infty$ except possibly for a set $E$ of $r$ of finite linear measure. A meromorphic function $a$ is said to be a small function of $f$ provided that $T(r, a)=S(r, f)$. We say that two non-constant meromorphic functions $f$ and $g$ share the value or small function $a \mathrm{CM}$ (counting multiplicities), if $f$ and $g$ have the same $a$-points with the same multiplicity.

In [1] R. Brück proved the following theorem:

THEOREM A. Let $f$ be a non-constant entire function satisfying $N\left(r, 1 / f^{\prime}\right)=$ $S(r, f)$. If $f$ and $f^{\prime}$ share the value $1 C M$, then $f-1=c\left(f^{\prime}-1\right)$, where $c$ is a nonzero constant.

It is asked naturally whether the value 1 of Theorem A can be simply replaced by small function $a(\not \equiv 0, \infty)$. We make an example which shows that the answer of this question is negative.

EXAmPle 1. Let $f(z)=1+\exp \left(e^{z}\right)$ and $a(z)=1 /\left(1-e^{-z}\right)$, by Lemma 1 , we know that $a$ is a small function of $f$. It is easy to see that $f$ and $f^{\prime}$ share $a \mathrm{CM}$ and $N\left(r, 1 / f^{\prime}\right)=0$, but $f-a \neq c\left(f^{\prime}-a\right)$, for every nonzero constant $c$. Indeed, $f-a=e^{-z}\left(f^{\prime}-a\right)$.

Keywords: Nevanlinna theory, uniqueness theorem, share CM, small function.

Classification Categories: Math. Subject classification: $30 \mathrm{D} 35$.

Received April 16, 2003; revised March 16, 2004. 
In this paper we shall generalize the result in Theorem A and obtain the following theorem:

THEOREM 1. Let $f$ be a non-constant entire function satisfying $N\left(r, 1 / f^{\prime}\right)=$ $S(r, f)$ and let $a(\not \equiv 0, \infty)$ be a meromorphic small function of $f$. If $f$ and $f^{\prime}$ share a $C M$, then $f-a=(1-k / a)\left(f^{\prime}-a\right)$, where $1-k / a=e^{\beta}, k$ is a constant and $\beta$ is an entire function.

From Theorem 1, we immediately deduce the following corollary:

COROLlaRY 1. Let $f$ be a non-constant entire function satisfying $N\left(r, 1 / f^{\prime}\right)=S(r, f)$ and let $a(\not \equiv 0, \infty)$ be an entire small function of $f$. If $f$ and $f^{\prime}$ share a $C M$, then either $f=f^{\prime}$ or $a \equiv$ const and $f-a=c\left(f^{\prime}-a\right)$, where $c(\neq 0,1)$ is a constant.

It is obvious that Theorem A is a special case of Theorem 1 or Corollary 1.

Remark 1. From Theorem 1, it is easy to see that $f(z)=$ $A \exp \left(\int(1-k / a(z))^{-1} d z\right)+k$, where $1-k / a(z)=e^{\beta(z)}, A(\neq 0), k$ are constants and $\beta$ is an entire function. This result includes Example 1 as a special case.

\section{Some lemmas}

For the proof of our results we need the following lemmas:

Lemma $1[2$, p. 50]. Let $f$ and $g$ be two transcendental entire functions. Then

$$
\lim _{r \rightarrow \infty} \frac{T(r, g)}{T(r, f(g))}=0 .
$$

Lemma $2\left[4\right.$, p. 96]. Let $f_{j}(j=1,2,3,4)$ be meromorphic functions and $f_{k}(k=1,2)$ are non-constants satisfying $\sum_{j=1}^{4} f_{j} \equiv 1$. If

$$
\sum_{j=1}^{4} N\left(r, \frac{1}{f_{j}}\right)+3 \sum_{j=1}^{4} \bar{N}\left(r, f_{j}\right)<(\lambda+o(1)) T\left(r, f_{k}\right) \quad(r \in I, k=1,2),
$$

where $\lambda<1$ and $I$ is a set of infinite measure. Then $f_{3} \equiv 1, f_{4} \equiv 1$, or $f_{3}+f_{4} \equiv 1$.

Lemma 3 [2]. Let $f$ be a non-constant meromorphic function, and $a_{1}, a_{2}, a_{3}$ be distinct small functions of $f$, then

$$
T(r, f) \leq \sum_{j=1}^{3} \bar{N}\left(r, \frac{1}{f-a_{j}}\right)+S(r, f) .
$$




\section{Proof of theorem 1}

From Theorem A, we know that Theorem 1 is valid for $a$ is a nonzero constant. Next we suppose that $a$ is a non-constant meromorphic function.

Since $f$ and $f^{\prime}$ share $a \mathrm{CM}$, there is an entire function $\beta$ such that

$$
f-a=e^{\beta}\left(f^{\prime}-a\right) \text {. }
$$

We claim that $T\left(r, e^{\beta}\right)=S(r, f)$. Differentiating (3.1) we obtain

$$
\left(\frac{a}{a^{\prime}} \beta^{\prime}+1\right) e^{\beta}+\frac{1}{a^{\prime}} f^{\prime}-\frac{\beta^{\prime} e^{\beta}}{a^{\prime}} f^{\prime}-\frac{e^{\beta}}{a^{\prime}} f^{\prime \prime} \equiv 1 .
$$

In order that applying Lemma 2 to (3.2), we consider the following two cases:

CASE I. $\quad\left(\left(a / a^{\prime}\right) \beta^{\prime}+1\right) e^{\beta} \equiv c$, where $c$ is a constant.

If $c=0,\left(a / a^{\prime}\right) \beta^{\prime}+1 \equiv 0$. By integration, we get $a=A e^{-\beta}$, where $A$ is a nonzero constant, and hence $T\left(r, e^{\beta}\right)=S(r, f)$. We also see that, if $c \neq 0$, $T\left(r, e^{\beta}\right)=S(r, f)$.

CASE II. $\left(1 / a^{\prime}\right) f^{\prime} \equiv$ const.

Then $T\left(r, f^{\prime}\right)=S(r, f)$. It follows that $N(r, 1 /(f-a))=N\left(r, 1 /\left(f^{\prime}-a\right)\right)=$ $S(r, f)$, and

$$
\begin{aligned}
m\left(r, \frac{1}{f-a}\right) & \leq m\left(r, \frac{f^{\prime}-a^{\prime}}{f-a}\right)+m\left(r, \frac{1}{f^{\prime}-a^{\prime}}\right) \\
& \leq T\left(r, f^{\prime}\right)+S(r, f)=S(r, f) .
\end{aligned}
$$

Thus, we have $T(r, f)=S(r, f)$ which is a contradiction.

Now suppose $\left(\left(a / a^{\prime}\right) \beta^{\prime}+1\right) e^{\beta}$ and $\left(1 / a^{\prime}\right) f$ are non-constants. Note that

$$
\begin{aligned}
N\left(r, \frac{1}{f^{\prime \prime}}\right) & \leq N\left(r, \frac{f^{\prime}}{f^{\prime \prime}}\right)+N\left(r, \frac{1}{f^{\prime}}\right) \leq T\left(r, \frac{f^{\prime \prime}}{f^{\prime}}\right)+N\left(r, \frac{1}{f^{\prime}}\right)+O(1) \\
& \leq 2 N\left(r, \frac{1}{f^{\prime}}\right)+\bar{N}(r, f)+S(r, f)=S(r, f) .
\end{aligned}
$$

Applying Lemma 2 to (3.2), we divide into the following three cases:

CASE $1 . \quad-\left(\beta^{\prime} e^{\beta} / a^{\prime}\right) f^{\prime} \equiv 1$.

Substituting this into (3.2) gives

$$
\frac{f^{\prime \prime}}{a \beta^{\prime}+a^{\prime}}-\frac{e^{-\beta} f^{\prime}}{a \beta^{\prime}+a^{\prime}} \equiv 1 \text {. }
$$

From this and the second fundamental theorem for $H=f^{\prime \prime} /\left(a \beta^{\prime}+a^{\prime}\right)$ 


$$
\begin{aligned}
T(r, H) \leq & N\left(r, \frac{1}{H}\right)+N\left(r, \frac{1}{H-1}\right)+\bar{N}(r, H)+S(r, H) \\
\leq & N\left(r, \frac{1}{f^{\prime \prime}}\right)+N\left(r, a \beta^{\prime}+a^{\prime}\right)+N\left(r, \frac{1}{f^{\prime}}\right)+N\left(r, a \beta^{\prime}+a^{\prime}\right) \\
& +\bar{N}\left(r, f^{\prime \prime}\right)+\bar{N}\left(r, \frac{1}{a \beta^{\prime}+a^{\prime}}\right)+S(r, f) \\
\leq & N\left(r, \frac{1}{f^{\prime \prime}}\right)+N\left(r, \frac{1}{f^{\prime}}\right)+\bar{N}\left(r, f^{\prime \prime}\right) \\
& +3\left(T(r, a)+T\left(r, a^{\prime}\right)+T\left(r, \beta^{\prime}\right)\right)+S(r, f)=S(r, f) .
\end{aligned}
$$

It follows that $T\left(r, f^{\prime \prime}\right)=S(r, f)$, and so

$$
\begin{aligned}
T\left(r, f^{\prime}\right) & =T\left(r, \frac{f^{\prime}}{f^{\prime \prime}} \cdot f^{\prime \prime}\right) \leq T\left(r, \frac{f^{\prime}}{f^{\prime \prime}}\right)+T\left(r, f^{\prime \prime}\right) \\
& \leq T\left(r, \frac{f^{\prime \prime}}{f^{\prime}}\right)+T\left(r, f^{\prime \prime}\right)+O(1) \\
& \leq T\left(r, \frac{f^{\prime \prime}}{f^{\prime}}\right)+S(r, f)=S(r, f),
\end{aligned}
$$

giving a contradiction.

CASE 2. $-\left(e^{\beta} / a^{\prime}\right) f^{\prime \prime} \equiv 1$.

Similarly as the Case 1 , we arrive at a contradiction.

CASE 3. $-\left(\beta^{\prime} e^{\beta} / a^{\prime}\right) f^{\prime}-\left(e^{\beta} / a^{\prime}\right) f^{\prime \prime} \equiv 1$.

Substitution of this identical equation in (3.2) gives

$$
f^{\prime}=-\left(a \beta^{\prime}+a^{\prime}\right) e^{\beta} \text {. }
$$

Differentiating (3.3) we find that

$$
f^{\prime \prime}=-e^{\beta}\left(a^{\prime \prime}+2 a^{\prime} \beta^{\prime}+a \beta^{\prime \prime}+a \beta^{2}\right) .
$$

Substituting (3.3) and (3.4) into above identical equation gives

$$
e^{-2 \beta}=\frac{2 a^{\prime} \beta^{\prime 2}}{a^{\prime}}+3 \beta^{\prime}+\frac{a}{a^{\prime}} \beta^{\prime \prime}+\frac{a^{\prime \prime}}{a^{\prime}} .
$$

This implies that $T\left(r, e^{\beta}\right)=S(r, f)$, and this proves the claim.

Now (3.1) can be written

$$
f^{\prime}=e^{-\beta}(f-b),
$$

where $b=a\left(1-e^{\beta}\right)$ is a small function of $f$. Since $N\left(r, 1 / f^{\prime}\right)=S(r, f)$, we see from (3.5) that

$$
N\left(r, \frac{1}{f-b}\right)=S(r, f) .
$$


From (3.6) and the second fundamental theorem for $F=f-b$

$$
\begin{aligned}
T(r, F) & \leq N\left(r, \frac{1}{F}\right)+N\left(r, \frac{1}{F-1}\right)+\bar{N}(r, F)-N\left(r, \frac{1}{F^{\prime}}\right)+S(r, F) \\
& \leq N\left(r, \frac{1}{F-1}\right)-N\left(r, \frac{1}{F^{\prime}}\right)+S(r, f) \\
& \leq T(r, F)-N\left(r, \frac{1}{F^{\prime}}\right)+S(r, f) .
\end{aligned}
$$

It follows that

$$
N\left(r, \frac{1}{f^{\prime}-b^{\prime}}\right)=S(r, f)
$$

From (3.7) and Lemma $3\left(a_{1}=0, a_{2}=b^{\prime}, a_{3}=\infty\right)$, we deduce that if $b^{\prime} \not \equiv 0$,

$$
T\left(r, f^{\prime}\right) \leq \bar{N}\left(r, \frac{1}{f^{\prime}}\right)+\bar{N}\left(r, \frac{1}{f^{\prime}-b^{\prime}}\right)+\bar{N}\left(r, f^{\prime}\right)+S\left(r, f^{\prime}\right)=S(r, f),
$$

which is a contradiction. Therefore, we have $b^{\prime} \equiv 0$ and so $a\left(1-e^{\beta}\right) \equiv k$, where $k$ is a constant. Combining with (3.1), we get $f-a=(1-k / a)\left(f^{\prime}-a\right)$.

Acknowledgement. I am grateful to the referee for valuable suggestion and comments.

\section{REFERENCES}

[ 1 ] BRÜCK, R., On entire functions which share one value CM with their first derivative, Results in Math., 30 (1996), 21-24.

[2] Hayman, W. K., Meromorphic functions, Clarendon Press, Oxford, 1964.

[3] Nevanlinna, R., Le théorème de Picard-Borel et la théorie des functions méromorphes, Gauthiers-Villars, Paris, 1929.

[4] Yi, H. X. ANd Yang, C. C., Uniqueness theory of meromorphic functions, Science Press, Beijing, 1995.

Department of Mathematics

Faculty of Education, AL-Nadira City

Ibb Province, P.O. Box No. 75508

REPUBLIC OF YeMEN 